\title{
Role of sepsis in delayed mortality
}

\author{
Amanpreet Kaur, Mitchell M. Levy \\ Division of Pulmonary and Critical Care Medicine, Alpert Medical School at Brown University, Providence, Rhode Island, USA \\ Correspondence to: Mitchell M. Levy, MD. Division of Pulmonary and Critical Care Medicine, Alpert Medical School at Brown University, \\ Providence, Rhode Island, USA. Email: mitchell_levy@brown.edu.
}

Submitted Aug 02, 2016. Accepted for publication Aug 08, 2016.

doi: 10.21037/atm.2016.08.24

View this article at: http://dx.doi.org/10.21037/atm.2016.08.24

Sepsis is defined as a clinical syndrome consisting of physiologic, pathologic and biochemical abnormalities due to a dysregulated inflammatory response to an infection (1). Over the last thirty years, sepsis has become one of the leading causes of hospital admissions as well as healthcare expenditure. The rates of sepsis in the US have increased dramatically. As demonstrated by an analysis of discharge records in US hospitals by the Agency of Healthcare Research and Quality (AHRQ) published in 2011, sepsis accounted for an estimated 1,665,000 hospitalizations in 2009, steadily increasing from the early 1990s (2). In 2011 alone, sepsis was reported to be the most expensive reason for hospitalization, resulting in more than $\$ 20$ billion (5.2\%) of total hospital costs (3).

Over the last two decades, implementation of aggressive sepsis education and awareness campaigns through the Surviving Sepsis Campaign, with a focus on early diagnosis and management, has resulted in documented decrease in in-hospital mortality of these patients (4). However, more recent studies have noted that the longer-term morbidity and mortality in patients who have survived sepsis remains high. Although not limited to sepsis survivors, recent data suggests that over half of ICU survivors suffer from cognitive impairment (5), up to $20 \%$ suffer from posttraumatic stress disorder (6), and approximately a third suffer from depression (7). Exercise limitation and decreased physical quality of life has also been reported in longitudinal follow up of ICU survivors (8).

Currently, there is conflicting evidence on etiology of the longer-term mortality after sepsis. It is unclear whether it is the patient's underlying comorbidities or the episode of sepsis itself that serves as the driving force behind the elevated long-term mortality.

In their recently published observational cohort study in the $B M \mathcal{F}$, Prescott and colleagues aimed to evaluate the late mortality, defined as 31 days to 2 years, attributable to sepsis rather than merely to other comorbid conditions and sociodemographic factors (9). Subjects for the study were obtained through the US Health and Retirement Study (HRS) and included Medicare beneficiaries $\geq 65$ years old who were surveyed at least once between 1998 and 2008 . The authors performed a series of propensity adjusted comparisons between four study cohorts while adjusting for obvious confounding factors, including sociodemographic status, comorbid conditions and baseline functional capacity. The four study cohorts included the primary cohort of patients admitted to the hospital with sepsis, and comparison cohorts of patients not in the hospital, patients in the hospital with inflammatory but non-infective disease, and patients in the hospital with infection but non-septic disease.

The researchers found a significantly increased burden of sepsis-associated mortality that persists for up to at least 2 years. Compared to the patients admitted to the hospital with a non-sepsis infection, patients with sepsis had a $10 \%$ absolute increase in late mortality. Furthermore, there was a $16 \%$ absolute increase in late mortality among sepsis patients when compared to those with sterile inflammatory conditions and a $22 \%$ absolute increase in late mortality when sepsis patients were compared to similar nonhospitalized elderly adults.

Further detailed analysis of patient subgroups also revealed that late mortality in the sepsis cohort seemed to be constant across subgroups defined by source of sepsis, age, sex, sociodemographic status, prior comorbidities and functional limitation. This particular finding strongly suggests that it is the episode of sepsis itself that serves as the driving force behind long term mortality. It should be 
noted that the higher the number of organ dysfunctions in sepsis, higher the mortality in this cohort. As the authors accurately point out, their findings do not argue against the important contribution of co-morbidity to the increased long-term mortality in sepsis. However, their data do argue for sepsis itself adding additional risk of long-term mortality in these patients. It is interesting that the higher mortality in the sepsis cohort $v s$. the group not admitted to hospital was present for the full 2-year follow up period, while the mortality difference in sepsis $v s$. admitted the hospital with non-sepsis infection only persisted for 1 year. One could argue that this further adds to the evidence that sepsis of any kind, even without organ failure, confers a higher risk of long term ( $>1$ year) mortality. Additionally, 30- and 90-day readmission rates were also noted to be higher in the sepsis cohort. This further adds to the financial impact of long term care for patients who are admitted for sepsis. The most common terminal diagnosis across all cohorts seemed to be associated with an infection, accounting for $22-30 \%$ of all deaths and which, as the authors point out, suggests a possible role of sepsis-related immune suppression in these recurrent infections and readmissions.

Overall, this was a robust, well-done, observational study. Prescott and colleagues appropriately adjusted for previously known confounding factors in this patient population and also identified plausible secondary cohort groups to compare with the primary cohort of sepsis patients. One of the study limitations is also its strength. The study population was limited to patients $>65$ years old due to the limitation of the database used for analysis. This limits the generalizability of the results to a younger population with sepsis. However, as the patient population in our ICUs is getting older, this study identifies an important, growing population and raises several important ideas that will foster further research in sepsis survivorship.

Several questions remain unanswered by this study; is there a specific phenotype of immunosuppressed or physiologically frail patient that is more likely to be affected by the increased risk of late mortality? Additionally, the mechanism(s) behind this late mortality risk remains unclear. As the authors mention, the study does raise the exciting possibility of finding interventions that can be taken to ameliorate this risk of long-term mortality in sepsis.

With the focus on sepsis research on early diagnosis and management over the last two decades, future research must address the long term care needs of patients who survive an episode of sepsis, with a focus on preservation of physiologic and functional capacity in these patients in order prevent acute and long term sequelae that result in readmissions and mortality after their episode of sepsis.

In recent years, the concept of post-ICU follow up clinics has received attention in the field of critical care medicine. Although this idea has been better received in the United Kingdom and Australia with numerous postICU follow up clinics opening in the last 15 years (10), several pulmonary and critical care divisions in the U.S have recently established their critical care recovery clinics $(11,12)$. The data presented in this study also underscores the importance of educating the primary care providers who are currently following up these patients in the outpatient setting regarding the increased late mortality risk of these sepsis survivors despite their ostensible resilience. In the near future, post-ICU follow up of survivors of sepsis may provide insight into the pathophysiology underlying the increase late mortality after sepsis which then may lead to interventions that ameliorate this risk.

\section{Acknowledgements}

None.

\section{Footnote}

Provenance: This is a Guest Editorial commissioned by Section Editor Zhi Mao, MD (Department of Critical Care Medicine, Chinese People's Liberation Army General Hospital, Beijing, China).

Conflicts of Interest: The authors have no conflicts of interest to declare.

Comment on: Prescott HC, Osterholzer JJ, Langa KM, et al. Late mortality after sepsis: propensity matched cohort study. BMJ 2016;353:i2375.

\section{References}

1. Shankar-Hari M, Phillips GS, Levy ML, et al. Developing a new definition and assessing new clinical criteria for septic shock: for the third international consensus definitions for sepsis and septic shock (Sepsis-3). JAMA 2016;315:775-87.

2. Elixhauser A, Friedman B, Strages E. Septicemia in US Hospitals, 2009. HCUP Statistical Brief \#122. Available online: http://www.hcup-us.ahrq.gov/reports/statbriefs/ sb122.pdf

3. Singer M, Deutschman CS, Seymour CW, et al. The 
Third International Consensus Definitions for Sepsis and Septic Shock (Sepsis-3). JAMA 2016;315:801-10.

4. Stevenson EK, Rubenstein AR, Radin GT, et al. Two decades of mortality trends among patients with severe sepsis: a comparative meta-analysis*. Crit Care Med 2014;42:625-31.

5. Pandharipande PP, Girard TD, Jackson JC, et al. Longterm cognitive impairment after critical illness. N Engl J Med 2013;369:1306-16.

6. Davydow DS, Gifford JM, Desai SV, et al. Posttraumatic stress disorder in general intensive care unit survivors: a systematic review. Gen Hosp Psychiatry 2008;30:421-34.

7. Jackson JC, Pandharipande PP, Girard TD, et al. Depression, post-traumatic stress disorder, and functional disability in survivors of critical illness in the BRAIN-ICU study: a longitudinal cohort study. Lancet Respir Med 2014;2:369-79.

Cite this article as: Kaur A, Levy MM. Role of sepsis in delayed mortality. Ann Transl Med 2016;4(19):378. doi: 10.21037/ atm.2016.08.24
8. Herridge MS, Tansey CM, Matté A, et al. Functional disability 5 years after acute respiratory distress syndrome. N Engl J Med 2011;364:1293-304.

9. Prescott HC, Osterholzer JJ, Langa KM, et al. Late mortality after sepsis: propensity matched cohort study. BMJ 2016;353:i2375.

10. Griffiths JA, Barber VS, Cuthbertson BH, et al. A national survey of intensive care follow-up clinics. Anaesthesia 2006;61:950-5.

11. Khan BA, Lasiter S, Boustani MA. CE: critical care recovery center: an innovative collaborative care model for ICU survivors. Am J Nurs 2015;115:24-31; quiz 34, 46.

12. Stollings JL, Huggins EL, Jackson JC, et al. It takes a team: contributions of each team member in a postintensive care syndrome clinic model. SCCM Critical Connections: Post-Intensive Care syndrome, 2015, 4 August. 